\title{
The AGREE Reporting Checklist: a tool to improve reporting of clinical practice guidelines
}

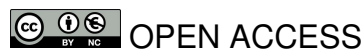

This Research Methods and Reporting article (BMJ 2016;352:i1152, doi:10.1136/bmj.i1152) was mistakenly published without an open access licence initially online and in the May 2016 academic print issue, and should have been published under the CC BY NC licence. The online version has since been corrected. 